\title{
LA REGULACIÓN DE LA SUCESIÓN, LOS PACTOS MATRIMONIALES Y LAS INCOHERENCIAS ENTRE LAS NORMAS DE DERECHO INTERNACIONAL PRIVADO EUROPEO ${ }^{1 *}$
}

\author{
Stefano Deplano \\ Profesor asociado en la Universidad de la Campania \\ E-mai: stefano.deplano@unicampania.it
}

\begin{abstract}
RESUMEN: El presente capítulo pretende centrarse en una cuestión problemática: la ley aplicable a los pactos entre cónyuges o parejas registradas transfronterizas relativos a la sucesión de la herencia del cónyuge o de la pareja registrada fallecida. El análisis comienza (sección 2) con un examen de la amplia definición de "acuerdo sucesorio" establecida en el Reglamento de sucesiones. En segundo lugar, se centra en algunos aspectos críticos del Reglamento sobre sucesiones que parecen complicar la planificación sucesoria de las parejas transfronterizas. Aunque el considerando $7 \mathrm{del}$ Reglamento sobre sucesiones establece que uno de los principales objetivos del Reglamento es que "los ciudadanos puedan organizar su sucesión", y su considerando 38 especifica que "el Reglamento sobre sucesiones debe permitir a los ciudadanos organizar su sucesión por adelantado eligiendo la ley aplicable a su sucesión", hay numerosas normas que no parecen estar en consonancia con este objetivo. Estas cuestiones se destacan en las secciones 3 y 4. Se presta especial atención (sección 5) a los acuerdos entre cónyuges o parejas registradas transfronterizas para planificar su sucesión. Por último (sección 6), se ofrecen algunas observaciones finales sobre el proceso de integración europea realizado a través del Derecho internacional privado (en adelante, DIP) y el papel de los tribunales en un sistema jurídico unitario y complejo.
\end{abstract}

Palabras clave: Reglamento sucesorio, "acuerdo sucesorio", ley aplicable, cónyuges, parejas registradas.

ABSTRACT: This paper intends to focus on a problematic issue: the law applicable to agreements between cross-border spouses or registered partners relating to succession of the estate of a deceased spouse or registered partner. The analysis begins (Section 2) with an examination of the broad definition of 'agreement as to succession' set out in the Succession Regulation. Secondly, it focuses on some critical aspects of the Succession Regulation that seem likely to complicate the succession planning of cross-border couples.

1 * Traducido por Fátima Pérez Ferrer, profesora titular de Derecho penal de la Universidad de Almería. 


\begin{abstract}
Although Recital 7 of the Succession Regulation establishes that one of the primary purposes of the regulation is that 'citizens must be able to organise their succession in advance', and its Recital 38 specifies that '[the Succession Regulation] should enable citizens to organise their succession in advance by choosing the law applicable to their succession', there are numerous rules that do not appear to be in line with this aim. These issues are highlighted in Sections 3 and 4. Particular attention is devoted (Section 5) to agreements between cross-border spouses or registered partners in order to plan their succession. Lastly (Section 6), some concluding remarks are offered on the process of European integration achieved through the private international law (PIL) and the role of the courts in a unitary and complex legal system.
\end{abstract}

Keywords: Succession Regulation, 'agreement as to succession', applicable law, cross-border spouses, registered partners.

\begin{abstract}
SUMARIO: I. INTRODUCCIÓN. II. COMPRENSIÓN DEL “PACTO SUCESORIO” Y SU RELACIÓN CON LOS INSTRUMENTOS NACIONALES. III. CUESTIONES PROBLEMÁTICAS RELACIONADAS CON LOS PACTOS SUCESORIOS DE VARIAS PERSONAS.

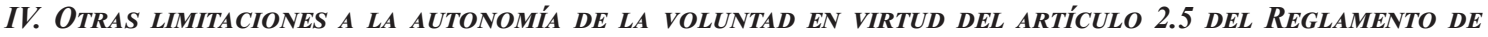

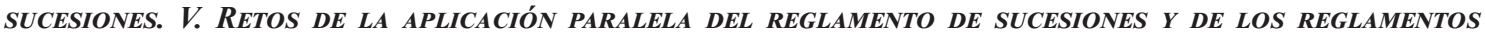
GEMELOS VI. OBSERVACIONES FINALES: ¿QUÉ LECCIONES SE PUEDEN EXTRAER DEL DEBATE ANTERIOR?
\end{abstract}

\title{
I. INTRODUCCIÓN
}

Desde mediados del siglo XIX, los juristas han sugerido la introducción de normas uniformes sobre el conflicto de leyes en materia de sucesiones ${ }^{2}$. Sin embargo, ha tenido que pasar más de un siglo para que las disposiciones jurídicamente vinculantes entraran finalmente en vigor.

Desde la Segunda Guerra Mundial, se ha producido una convergencia gradual del Derecho internacional privado en ámbitos en los que la segmentación de las normas nacionales era un obstáculo para la integración ${ }^{3}$. Centrándonos en el derecho de sucesiones, este proceso se ha extendido a través de una serie de iniciativas como la Conferencia de La Haya de Derecho Internacional Privado 4 y las investigaciones realizadas por el Institut de droit international ${ }^{5}$ y el Groupe européen de droit international privé ${ }^{6}$.

2 V. SCIALOJA, Sistema del diritto romano attuale. Federico Carlo di Savigny. Traduzione dall'originale tedesco, vol. 8, Unione Tipografico-Editrice, Turín 1898, pp. 115-129.

3 J. BONELL, "Comparazione giuridica e unificazione del diritto", en AA.VV. (eds.), Diritto privato comparato. Istituti e problemi, $4^{a}$ ed., Laterza, Roma-Bari 2011, p. 3 y ss. también para otras referencias bibliográficas.

4 E. RABEL, 'The Conflict of Laws: A Comparative Study' [1958] Michigan Legal Studies Series, p. 250 y ss. y H. LEWALD, Questions de droit international des successions. Recueil de recours [1925] La Haya 9, p. 5 y ss.

5 H. WEHBERG (ed.), Résolutions de l'Institut de droit international (1873-1956), Basilea 1957, p. 40 y ss.

6 Véase "The Proposal for a Convention concerning jurisdiction and the enforcement of judgments in family and succession matters of 1993": www.gedip-egpil.eu/documents/gedip-documents-3pe.html (consultado el 
En el plano legislativo, cabe mencionar en particular el Convenio de 5 de octubre de 1961 sobre los conflictos de leyes relativos a la forma de las disposiciones testamentarias $^{7}$ y el Convenio de La Haya de 1 de agosto de 1989 sobre la ley aplicable a las sucesiones por causa de muerte ${ }^{8}$. Aunque este último no ha entrado nunca en vigor, ha sido un importante punto de referencia para varios legisladores nacionales y, sobre todo, en la elaboración de las normas europeas de conflicto de leyes en materia de sucesiones ${ }^{9}$, es decir, el Reglamento sobre sucesiones (también conocido como Reglamento Bruselas IV) ${ }^{10}$.

El proceso de aprobación de este instrumento legislativo estuvo marcado por considerables dificultades. Éstas se debieron principalmente a las diferentes tradiciones jurídicas de los Estados miembros, especialmente arraigadas en el ámbito del derecho de sucesiones ${ }^{11}$. Por ello, no es de extrañar que el diálogo entre los juristas teóricos y prácticos que participaron en este ejercicio de armonización fuera especialmente difícil ${ }^{12}$.

Excepto en Dinamarca e Irlanda ${ }^{13}$, el Reglamento de sucesiones se aplica en todos los países de la UE. Este Reglamento es importante por varias razones.

En primer lugar, el Reglamento de sucesiones ha supuesto una "revolución"14 en los sistemas jurídicos de varios Estados miembros de la UE. Ello se debe a que el Regla-

29.06.2021). Véase también E. JAYME, “Entwurf eines EG-Familienund Erbrechtsübereinkommens” [1994] Praxis des Internationalen Privat-und Verfahrensrechts 14, p. 67 y ss.

7 F. MAJOROS, Les conventions internationales en matière de droit privé. Abrégé théoretique et traité pratique, vol. 2, Pedone, París 1976, p. 395.

8 P. LAGARDE, 'La nouvelle convention de La Haye sur la loi applicable aux successions [1989] Revue critique de droit international privé 78, p. 249; H. VAN LOON, 'The Hague Convention on the Law Applicable to Succession to the Estates of Deceased Persons' [1989] Hague Yearbook of International Law, p. 48; A. BORRAS, 'La convention de la Haye de 1989 sur la loi applicable aux successions à cause de mort et l'Espagne', en A. BORRAS, A. BUCHER, T. STRUYCKEN, M. VERWILGHEN (eds.), E Pluribus Unum. Liber Amicorum Georges A.L. Droz, Martinus Nijhoff Publishers, La Haya-Boston-Londres 1996, p. 7. Analiza las razones de la falta de éxito del Convenio de La Haya T. PERTOT, "Certificado sucesorio europeo", en M.J. CAZORLA GONZÁLEZ, M. GIOBBI, J. KRAMBERGER ŠKERL, L. RUGGERI y S. WINKLER (eds.) Property relations of cross border couples in the European Union, ESI, Nápoles, 2020, p. 124 y ss.

9 Sobre este punto, véase M. PAZDAN y M. ZACHARIASIEWICZ, "Highlights and Pitfalls of the EU Succession Regulation” [2020] Problemy Prawa Prywatnego Międzynarodowego 26, p. 127.

10 The Succession Regulation entered into force on 16.08.2012 and applies to successions opened on or after 17.08.2015.

11 A. DAVİ, "Introduction”, en A.L. CALVO CARAVACA, A. DAVİ y H.P. MANSEL (eds.), The EU Succession Regulation: A Commentary, Cambridge University Press, Cambridge 2016, p. 1 y ss; M. PFEIFFER, "Seguridad jurídica y previsibilidad en el Derecho internacional de sucesiones" [2016] Journal of Private International Law 12 , pp. 566-570.

12 J. HARRIS, "The proposed EU regulation on succession and wills: prospects and challenges" [2008] Trust Law International 22, p. 181; E. LEIN, "A further step towards a European Code of Private International Law: The Commission proposal for a Regulation on succession' [2009] Yearbook of Private International Law 11, p. 107; A. BONOMI y C. SCHMID (eds.), Successions internationales. Réflexions autour du futur règlement européen et son impact pour la Suisse, Ginebra, 2010; MAX PLANCK INSTITUTE, “Comments on the European Commission's Proposal for a Regulation of the European Parliament and of the Council on jurisdiction, applicable law, recognition and enforcement of decisions and authentic instruments in matters of succession and the creation of a European Certificate of Succession" [2010] Rabels Zeitschrift für ausländisches und internationales Privatrecht 74, p. 522.

13 De conformidad con los artículos 1 y 2 del Protocolo $n^{\circ} 21$ sobre la posición del Reino Unido respecto al espacio de libertad, seguridad y justicia, anejo al Tratado de la Unión Europea y al Tratado de Funcionamiento de la Unión Europea, este antiguo Estado miembro decidió no participar en la adopción del Reglamento y no estar sujeto a su aplicación.

14 P. LAGARDE, "Les principes de base du nouveau règlement européen sur les successions" [2012] Revue critique de droit international privé 101, p. 691: Este reglamento [...] constituye una verdadera revolución para el derecho francés actual; C. KOHLER, L'autonomie de la volonté en droit international privé: un principe universel entre 
mento europeo, una opción ampliamente aprobada por la doctrina jurídica ${ }^{15}$, ha adoptado el denominado sistema monista (ya presente en las normas de conflicto de leyes alemanas y austriacas), destinado a aplicar una única norma de conflicto de leyes a todos los bienes que componen una herencia y lograr así una sucesión unitaria ${ }^{16}$. En segundo lugar, el artículo 21 del Reglamento de sucesiones ha establecido la "residencia habitual"17 como criterio para identificar tanto la ley, como la jurisdicción aplicables en materia de sucesiones. En consecuencia, los Estados que utilizaban la nacionalidad del difunto como principal factor de vinculación (por ejemplo, Italia y Alemania) ${ }^{18}$ experimentan un importante cambio hacia un nuevo paradigma ${ }^{19}$. En tercer lugar, el número de decisiones prejudiciales ${ }^{20}$ y la jurisprudencia del $\mathrm{TJUE}^{21}$ el derecho relativo al Reglamento de sucesiones está en constante crecimiento. Por último, el Reglamento de sucesiones ha dado lugar a una importante evolución en los ordenamientos jurídicos nacionales ${ }^{22}$.

El presente capítulo pretende centrarse en una cuestión problemática: la ley aplicable a los pactos entre cónyuges o parejas registradas transfronterizas relativos a la sucesión de la herencia del cónyuge o de la pareja registrada fallecida. El análisis comienza (sección 2) con un examen de la amplia definición de "acuerdo sucesorio" establecida en

libéralisme et étatisme [2013] Recueil des Cours 359, p. 463: "Constituye un verdadero punto de inflexión copernicano para la materia".

15 A. DUTTA, "Succession and Wills in the Conflict of Laws on the Eve of Europeanisation" [2009] Rabels Zeitschrift für ausländisches und internationales Privatrecht 73, p. 555; A. BONOMI, "Choice of-Law Aspects of the Future EC Regulation in Matters of Succession-A First Glance at the Commission's Proposal", en K. BOELE-WOELKI, T. EINHORN, D. GIRSBERGER y S. SYMONIDES (eds.), Convergence and Divergence in Private International Law. Liber Amicorum Kurt Siehr, Eleven International Publishing, La Haya-Zurich 2010, p. 162; M. ZAŁUCKI, "Attempts to harmonize the inheritance law in Europe: past, present, and future" [2018] Iowa Law Review 103, p. 2330.

16 Para una perspectiva general, véase A. DAVÌ, supra n. 10, p. 3 y s.; A. BONOMI, “Succession”, en J. BASEDOW, G. RÜHL, F. FERRARI, P.A. DE MIGUEL ASENSIO (eds.), Encyclopedia of private in-ternational law, Elgar Publishing, Cheltenham 2017, p. 1683 y ss.

17 Sobre este punto, véase M. BOGDAN, 'The EC Treaty and the Use of Nationality and Habitual Resi-dence as Connecting Factors in International Family Law', en M. PERTEGÁS, G. STRAETMANS y F. SWENNEN (eds.), International Family Law for the European Union, Intersentia, Antwerp 2007, pp. 314-316. Véase también J. PIRRUNG, "Hague Conference on PIL", en J. BASEDOW, K.J. HOPT y R. ZIMMERMANN (eds.), The Max Planck Encyclopedia of European Private Law, vol. I, Oxford University Press, Oxford 2012, pp. 818-819; W. PINTENS, "Public Policy in Suc-cession Matters", en Liber Amicorum Kohler, Gieseking Verlag, Bielefeld 2018, p. 395 .

18 La LIF italiana, por ejemplo, utilizaba la nacionalidad como principal factor de conexión (artículo 46, Ley n ${ }^{\circ}$ 218 de 31 de mayo de 1995). Lo mismo ocurría en la LIF alemana (art. 25 I Einführungsgesetz BGB). Véase A. BONOMI, "Testamentary Freedom or Forced Heirship? Balancing Party Autonomy and the protection of Family Members', en M. ANDERSON y E. ARROYO I AMAYUELAS (eds.), The Law of Succession. Testamentary Freedom. European Perspectives, Europa Law Publishing, Groningen/Amsterdam 2011, p. 30.

19 M. PAZDAN y M. ZACHARIASIEWICZ, arriba n. 8, p. 127.

20 Entre las recientes solicitudes de cuestiones prejudiciales se encuentran: C-277/20 (véase, en detalle, la nota 42 infra); C-301/20 (validez y eficacia de un certificado de sucesión); C-387/20 (si la admisibilidad de una elección de ley en virtud del Reglamento prevalece sobre un acuerdo bilateral entre un Estado miembro y un tercero que no prevé la elección en materia de sucesión).

21 Por ejemplo, C-218/16 Kubicka, ECLI:EU:C:2017:755; C-558/16 Mahnkopf, ECLI:EU:C:2018:138 (véase más adelante n. 85); C-20/17 Oberle, ECLI:EU:C:2018:485; C-658/17 WB, ECLI:EU:C:2019:444; C-102/18 Brisch, ECLI:EU:C:2019:34; C-80/19 E.E., ECLI:EU:C:2020:569.

22 A. SANDERS, 'EU Formalities for Matrimonial Property Agreements and their effects on German Family Law', en J.M. SCHERPE y E. BARGELLI (eds.), The Interaction between Family Law, Succession Law and Private International Law. Adapting to change, Intersentia, Cambridge - Antwerp - Chicago 2021, p. 79 y ss.; E. GOOSENS, 'The Impact of the European Certificate of Succession on National Law. A Trojan Horse or Much Ado about Nothing”, en J.M. SCHERPE y E. BARGELLI (eds.), ibídem, p. 157 ss. 
el Reglamento de sucesiones. En segundo lugar, se centra en algunos aspectos críticos del Reglamento sobre sucesiones que parecen complicar la planificación sucesoria de las parejas transfronterizas. Aunque el considerando 7 del Reglamento sobre sucesiones establece que uno de los principales objetivos del Reglamento es que "los ciudadanos puedan organizar su sucesión por adelantado", y su considerando 38 especifica que "el Reglamento sobre sucesiones debe permitir a los ciudadanos organizar su sucesión por adelantado eligiendo la ley aplicable a su sucesión", hay numerosas normas que no parecen estar en consonancia con este objetivo. Estas cuestiones se destacan en las secciones 3 y 4. Se presta especial atención (sección 5) a los acuerdos entre cónyuges o parejas registradas transfronterizas para planificar su sucesión. Por último (sección 6), se ofrecen algunas observaciones finales sobre el proceso de integración europea realizado a través del Derecho internacional privado (en adelante, DIP) y el papel de los tribunales en un sistema jurídico complejo.

\section{ANÁLISIS DEL "PACTO SUCESORIO"Y SU RELACIÓN CON LOS INSTRUMENTOS NACIONALES}

Con una redacción similar a la del artículo 8 del Convenio de La Haya de 1989, el artículo 3, apartado 1, letra b), del Reglamento sobre sucesiones define el pacto sucesorio como un "todo acuerdo, incluido el resultante de testamentos recíprocos, por el que se confieran, modifiquen o revoquen, con o sin contraprestación, derechos relativos a la sucesión o las sucesiones futuras de una o más personas que sean partes en dicho acuerdo"23. Los acuerdos en materia de sucesión suelen ser contratos. El objetivo de esta norma es, por tanto, excluirlos de la aplicación del Reglamento Roma $\mathrm{I}^{24}$. En segundo lugar, la definición es totalmente independiente de las definiciones correspondientes establecidas en las legislaciones nacionales ${ }^{25}$, que tienden a ser mucho más amplias. Esta es una observación fundamental a los efectos de este capítulo: el citado artículo puede aplicarse a un gran número de instituciones jurídicas, incluidas las tradicionalmente excluidas del ámbito del Derecho de sucesiones, como los fideicomisos y, sobre todo, los acuerdos relativos a las relaciones matrimoniales ${ }^{26}$.

En primer lugar, no está claro si las disposiciones del capítulo III del Reglamento de sucesiones son aplicables tanto a los acuerdos por los que el testador dispone su propia sucesión en vida como a los acuerdos de sucesión testamentaria ${ }^{27}$ (por los que una persona

23 M. WELLER, “Art. 1”, en A.L. CALVO CARAVACO, A. DAVÌ y H.P. MANSEL (eds.), arriba n. 10, pp. 76 y ss.

24 Así lo señala G. BIAGIONI, “Art. 1”, en P. FRANZINA y F. SALERNO (eds.), Commentario al Regolamento n. 593/2008 del Parlamento europeo e del Consiglio del 17 giugno 2008 sulla legge applicabile alle obbligazioni contrattuali ("Roma I"), en Nuove leggi civili commentate, Padua 2009, p. 568, donde se sostiene que los pactos sucesorios se rigen por la lex successionis.

25 A. KÖHLER, "Agreements as to Succession Under the New European Private International Law" [2005] Revija za evropsko parvo, pp. 25-30; L.E. PERRIELLO, "Succession agreements and public policy within EU Regulation 650/2012", en S. LANDINI (ed.), Insights and proposals related to the application of the European Succession Regulation 650/2012, ESI, Nápoles 2019, p. 375; A. DUTTA, "Article 3" in Münchener Kommentar zum Bürgerlichen Gesetzbuch, vol. 12, 8th ed., Beck, Múnich 2020, n 9 y ss.

26 Véase, más adelante, la sección 5.

27 Para el sistema jurídico italiano, véase C. GANGI, La successione testamentaria nel vigente diritto italiano, Giuffré, Milán 1964, p. 40 ss.; M.V. DE GIORGI, I patti sulle successioni future, Jovene, Nápoles 1976, p. 60 ss.; G. GROSSO y A. BURDESE, "Le successioni. Parte generale', en G. VASSALLI (ed.) Trattato di diritto civile, 
tiene derechos hereditarios derivados de una sucesión que aún no está en fase de sucesión), así como a los acuerdos de rechazo de la sucesión (negociados entre personas vivas, por los que una persona renuncia a los derechos que se derivarán de una futura sucesión) ${ }^{28}$.

Una interpretación literal de la letra b) del apartado 1 del artículo 3 del Reglamento sobre sucesiones hace ciertamente aplicable el Reglamento a los acuerdos ${ }^{29}$ por los que el testador dispone su propia sucesión en vida. En cambio, varios autores parten de la base de que el citado artículo no se aplica necesariamente a los pactos sucesorios testamentarios y a los pactos de rechazo de la sucesión, porque en estos casos la persona cuya sucesión es problemática no es parte del contrato. En consecuencia, existe una opinión generalizada de que los pactos sucesorios testamentarios y los pactos de rechazo de la sucesión, si se celebran en presencia de un elemento extranjero, deben regirse por la lex contractus (y no por la lex successionis) ${ }^{30}$. Este argumento es digno de aceptación, pero con una aclaración ${ }^{31}$ : si, en el caso concreto, la persona cuya sucesión está en juego es parte del pacto sucesorio, debe aplicarse el Reglamento de sucesiones. Esto se debe a que el acuerdo (de sucesión testamentaria o de rechazo de la sucesión) es, en concreto, parte de la planificación del patrimonio del fallecido.

También la doctrina italiana ha señalado recientemente que las normas aplicables a un pacto sucesorio no pueden prescindir de la finalidad concreta a la que sirve el pacto ${ }^{32}$ : siempre es necesario comprobar si el pacto sucesorio tiene por efecto la creación, modificación o extinción de los derechos sobre un futuro patrimonio de una persona que es "parte en el contrato", tal y como establece el artículo 3, apartado 1, del Reglamento de sucesiones. Este no es el caso, por ejemplo, de un acuerdo bilateral de rechazo de la sucesión en el que, por ejemplo, un hermano promete al otro renunciar a la futura herencia de su padre. Por lo tanto, además de la redacción del Reglamento de sucesiones, los tribunales deben referirse siempre a la finalidad concreta que pretende alcanzar un acuerdo específico (la llamada interpretación orientada a la función) ${ }^{33}$.

vol. XII, UTET, Turín 1977, p. 92 ss; L. FERRI, 'Successioni in generale - Artt. 456-511', en A. SCIALOJA y G. BRANCA (eds.), Commentario al codice civile, Zanichelli, Bolonia-Roma, 1980, p. 95 y ss.; M.V. DE GIORGI, Patto successorio, en Enc. dir., XXXII, Giuffré, Milán 1982, p. 533 y ss.

28 Para Bélgica, véase B. DELAHAYE y F. TAINMONT, Le rapport des donations à la lumière de la réforme du droit successoral. L'option, la condition, le terme et la substitution: effets civils et fiscaux sur l'organisation et la transmission d'un patrimoine, Limal, Anthemis 2017, p. 367. Para Francia, véanse los $\S \S 3$ y 4 infra, y G. RAOULCORMEIL, "La persistence de la prohibition des pactes successoraux" [2012] Les Petites Affiches, p. 25 y ss.

29 Con referencia al sistema jurídico alemán A. DUTTA, "Artículo 3", n. 24 anterior.

30 J. RODRÍGUEZ RODRIGO, “Art. 25”, en A.L. CALVO CARAVACO, A. DAVÌ y H.P. MANSEL (eds.), n. 10 supra, p. 382 y ss. abogando por la aplicación de la lex contractus; A. FUSARO, "Linee evolutive del diritto successorio europeo" [2014] Giustizia civile, pp. 510-538. En sentido contrario P. KINDLER, 'La legge applicabile ai patti successori nel Regolamento UE 650/2012' [2017] Rivista diritto internazionale privato e processuale, 2017, p. 17 considera que la amplia redacción del art. 3(1)(b) implica que también se aplica a los acuerdos de rechazo de la sucesión.

31 Como destaca, entre otros, L.E. PERRIELLO, "Succession agreements and public policy within EU Regulation 650/2012", supra n. 24, p. 377.

32 V. BARBA, I patti successori e il divieto di disposizione della delazione, ESI, Nápoles, 2015, p. 32 y ss. es crítico con la doctrina jurídica que tiende a uniformizar el alcance de los pactos sucesorios. Véase también F. MAGLIULO, "Il divieto del patto successorio istitutivo nella pratica negoziale" [1992] Rivista del notariato, p. 1418 y ss.; C. CACCAVALE, "Il divieto dei patti successori”, en P. RESCIGNO (ed.), "Successioni e donazioni”, vol. 1, CEDAM, Padua 1994, p. 25 y ss.; C. CACCAVALE, "Patti successori: il sottile confine tra nullità e validità negoziale" [1995] Notariato, p. 552 y ss.

33 Enfatizar las funciones en lugar de las estructuras en el derecho de sucesiones es la idea de P. PERLINGIERI, "La funzione sociale del diritto successorio" [2009] Rassegna di diritto civile, pp. 121-131, señalando que la 
Siguiendo esta perspectiva, hay quienes opinan que los contratos para hacer (o no hacer) un testamento, están también sujetos a la letra b) del apartado 1 del artículo 3 del Reglamento sobre sucesiones, opinión que puede considerarse digna de aceptación ${ }^{34}$. Estos contratos están muy extendidos en los sistemas de derecho anglosajón ${ }^{35}$. No confieren derechos sucesorios directos, sino que obligan a la parte cuyo patrimonio está en juego (no) a tomar una o varias disposiciones relativas a sus bienes después del fallecimiento. Aunque no están cubiertos por la disposición literal del artículo 3.1.b) del Reglamento de sucesiones, una interpretación orientada a la función lleva a aplicar también a esta proposición la norma mencionada en último lugar ${ }^{36}$.

Otro problema es la relación hermenéutica entre los artículos 3.1.b) y 1.2.g) del Reglamento de sucesiones. Esta última disposición excluye del ámbito de aplicación del Reglamento "los derechos de propiedad, los intereses y los bienes creados o transmitidos por medios distintos de la sucesión, por ejemplo, mediante donaciones [...]". En el caso de las donaciones mortis causa se plantean algunas cuestiones específicas ${ }^{37}$ : los estudiosos han subrayado que esta categoría de actos merece ser equiparada a los pactos sucesorios ${ }^{38}$. Se trata de un argumento que merece ser respaldado, ya que estas categorías de actos, en el plano funcional, persiguen el mismo objetivo que los pactos sucesorios: la planificación del patrimonio ${ }^{39}$.

Una interpretación orientada funcionalmente también lleva a considerar que la do-nation-partage o partage d'ascendant, l'institution contractuelle, la donation de biens à venir, y el llamado patto di famiglia (el primero típico del ordenamiento jurídico francés, el segundo italiano ${ }^{40}$ ) a pesar de tener las características típicas de un pacto sin

estructura no es un prius, sino un post con respecto a los efectos del acto; en consecuencia, no puede determinarse de antemano, sino sólo teniendo en cuenta los intereses perseguidos por la autonomía de la voluntad.

34 A. DAVİ y A. ZANOBETTI, n. 24, p. 68.

35 Véase R. KERRIDGE, 'Parry and Kerridge: The Law of Succession', 13ª ed, Sweet \& Maxwell, Londres 2016, pp. 110 y ss.

36 A favor de la inclusión de estos instrumentos en el ámbito de aplicación del artículo 25 del Reglamento de sucesiones: C. DÖBREINER, Das internationale Erbrecht nach der EU-Erbrechtsverordnung (Teil II), Mitteilungen des Bayerischen Notarvereins 2013, Múnich 2014, p. 439; A. DAVI y A. ZANOBETTI, Il nuovo diritto internazionale privato europeo delle successioni, Giappichelli, Turín 2014, p. 106; A. BONOMI, en A. BONOMI y P. WAUTELET, Le droit européen des successions, $2^{\mathrm{a}}$ ed., Bruylant, Bruselas 2016, pp. 157 y ss. Para el punto de vista opuesto: C.F. NORDMEIER, "Erbverträge und nachlassbezogene Rechtsgeschäfte in der EuErbVO-eine Begriffsklärung" [2013] Zeitschrift für Erbrecht und Vermögensnachfolge, p. 123.

37 Para la jurisprudencia italiana, véase, Cass., 29 de julio de 1971, n 2404, en Giustizia civile, 1971, I, p. 1536; Cass., 16 de febrero de 1995, $\mathrm{n}^{\circ}$ 1683, ibídem, 1995, I, p. 1501; Cass., 9 de mayo de 2000, $\mathrm{n}^{\circ}$ 5870, en Rivista del notariato, 2001, p. 227; Cass., 19 de noviembre de 2009, $\mathrm{n}^{\circ}$ 24450, en Nuova giurisprudenza civile commentata, 2010, 5, I, p. 560 y ss.

38 H. DÖRNER, "EuErbVO: Die Verordnung zum Internationalen Erb- und Erbverfahrensrecht ist in Kraft!". [2012] Zeitschrift für Erbrecht und Vermögensnachfolge, p. 508; A. DUTTA, "Das neue internationale Erbrecht der Europäischen Union - Eine erste Lektüre der Erbrechtsverordnung" [2013] Zeitschrift für das gesamte Familienrecht, pp. 5-10; P. LAGARDE, "Les principes de base du nouveau règlement européen sur les successions", n. 13 anterior, p. 717.

39 El Oberster Gerichtshof (Austria) planteó una cuestión prejudicial el 24 de junio de 2020 - asunto C-277/20. El Tribunal austriaco solicitó que se decidiera si la donación mortis causa constituye un pacto sucesorio y si el Reglamento se aplica a las elecciones de la ley aplicable realizadas antes del 17 de agosto de 2015.

40 P. KINDLER, "La legge applicabile ai patti successori nel regolamento UE nr 650/2012", n. 30 anterior, pp. 17-18; F. VISMARA, "Patti successori nel regolamento (UE) n. 65072012 e patti di famiglia: un'interferenza possibile?". [2014] Rivista di diritto internazionale privato e processuale, p. 813. Para el punto de vista contrario, D. DAMASCELLI, "Le pacte de famille", en A. BONOMI y M. STEINER (eds) Les pactes successoraux en droit comparé et en droit international privé, Libraire Droz, Genève 2008, p. 626. 
contraprestación deben regirse por el Reglamento de sucesiones si, sin duda, sirven para llevar a cabo la planificación patrimonial ${ }^{41}$.

\section{CUESTIONES PROBLEMÁTICAS RELACIONADAS CON LOS PACTOS SUCESORIOS DE VARIAS PERSONAS}

Tal y como se ha indicado en el apartado anterior, la definición de pacto sucesorio mencionada anteriormente afecta a varios ámbitos de los sistemas jurídicos nacionales. Además del problema de las definiciones, también es necesario analizar en detalle las normas aplicables a los acuerdos que contienen elementos internacionales. Estas normas se establecen en el artículo 25 del Reglamento de sucesiones, que distingue si el acuerdo tiene por objeto regular la sucesión de una o varias personas.

Este capítulo se centra en los pactos de sucesión de varias personas para poner de relieve cómo determinadas opciones adoptadas por el Reglamento de sucesiones pueden dificultar la planificación de la sucesión de quienes residen habitualmente en los Estados miembros de la $\mathrm{UE}^{42}$.

Tal y como se establece en el apartado 2 del artículo 25 del Reglamento de sucesiones, un pacto sucesorio relativo a la sucesión de varias personas sólo es admisible si se cumplen todas las leyes que, en virtud de dicho Reglamento, habrían regido la sucesión de todos los implicados si hubieran fallecido el día en que se celebró el pacto. Por lo que respecta a su validez material y a sus efectos vinculantes entre las partes, el pacto sucesorio se regirá por la ley con la que guarde mayor relación. La ley que regirá la sucesión regulará, por tanto, aspectos como $^{43}$ : la capacidad para realizar o recibir disposiciones de bienes en caso de fallecimiento; la admisibilidad de la representación; la interpretación de la disposición, y las cuestiones relativas al consentimiento o la intención de la persona que realiza la disposición ${ }^{44}$.

En la práctica, esto supone un problema importante, ya que las normas relativas a las limitaciones del poder de disposición derivadas del cumplimiento de las normas nacionales imperativas pueden no conocerse en su totalidad en el momento en que se celebra el pacto sucesorio. Cuanto mayor sea la distancia entre la fecha de celebración del pacto y la fecha de apertura de la sucesión, más grave será el problema. Este aspecto representa un primer e incisivo problema en cuanto a la seguridad en la planificación de

41 P. LAGARDE, "Les principes de base du nouveau règlement européen sur les successions", en U. BERGQUIST, D. DAMASCELLI, R. FRIMSTON, P. LAGARDE, F. ODERSKY Y B. REINHARTZ (eds.), EU Regulation on succession and wills. Commentary, Ottoschmidt, Colonia 2015, p. 148; C.F. NORDMEIER, "Die französische institution contractuelle im Internationalen Erbrecht: International-privatrechtliche und sachrechtliche Fragen aus deutscher und europäischer Perspektive" [2014] Praxis des Internationalen Privat- und Verfahrensrechts 34, p. 424-425; S. FRANK, D. BUREAU y H.M. WATT, Droit international privé, vol. 2, París 2017, p. 316; M. REVILLARD, Droit international privé et européen: pratique notariale, París 2018, p. 661.

42 Véase, a este respecto, el considerando 38 del Reglamento de sucesiones. Analiza la relación entre el Reglamento de sucesiones y los ordenamientos jurídicos de terceros países J. BASEDOW, "Member States" and "Third States” in the Succession Regulation' [2020] Problemy Prawa Prywatnego Międzynarodowego 26, p. 15 y ss.

43 D. DAMASCELLI, Diritto internazionale privato delle successioni a causa di morte (dalla 1. n. 218/1995 al Reg. UE n. 650/2012), Giuffré, Milan 2013, p. 96.

44 En relación con la aplicación de una norma interpretativa perteneciente al derecho del estado de Illi-nois v. Amtsgericht Hamburg-Wandsbeck, 17.05.2018 [2018] Zeitschrift für Das Gesamte Familienrecht, p. 1274 y ss. con un comentario crítico de Ludwig. 
la sucesión. Como ya se ha dicho, en el momento de celebrar el acuerdo, las partes no pueden tener ninguna certeza sobre la existencia de limitaciones: éstas dependen de una ley futura que no se puede determinar en ese momento y que es susceptible de cambios imprevisibles ${ }^{45}$. Esta incertidumbre sobre la ley que debe aplicarse es totalmente insatisfactoria, sobre todo si se tiene en cuenta el objetivo de "facilitar la aceptación de los derechos de sucesión adquiridos como consecuencia de un pacto sucesorio", tal como se indica en el considerando 49 del Reglamento sobre sucesiones.

Un elemento que aumenta la incertidumbre en la planificación de la sucesión son las normas sobre la llamada reserva hereditaria. Los sistemas jurídicos de varios Estados miembros (como los de Italia y Francia) limitan la autonomía del difunto reservando una parte de la herencia a determinadas categorías de herederos, denominados "herederos forzosos" (eredi necessari en Italia, héritiers réservataires en Francia). En presencia de estos herederos, la sucesión se divide en dos partes: la "porción disponible" puede darse libremente a cualquiera, la "porción reservada", en cambio, pertenece a los "herederos forzosos". Es evidente que las normas adoptadas por el legislador europeo no pretenden entrar en conflicto con las normas nacionales de protección de la reserva hereditaria ${ }^{46}$. No se trata de una posición revolucionaria: el Reglamento de sucesiones no se aparta ni del Convenio de La Haya de 1989 ni de las leyes de conflicto nacionales ${ }^{47}$, que, en cualquier caso, respetan los derechos de esa categoría particular de herederos ${ }^{48}$.

Este planteamiento parece reflejarse desde otra perspectiva en la propuesta original de la Comisión, cuando en el apartado 4 del artículo 18, correspondiente al artículo 25 del Reglamento sobre sucesiones, excluía a la parte implicada en el pacto sucesorio de la protección que le confería la lex successionis en ese momento ${ }^{49}$. El objetivo de la norma era evitar que la ley aplicable a la sucesión limitara la validez del pacto sucesorio. Sin embargo, esta disposición se suprimió del texto final del Reglamento. Como confirma el considerando 50 del Reglamento sobre sucesiones, una parte de un pacto sucesorio puede pretender, aún a costa de infringir la norma general nemo potest venire contra factum proprium, que "los efectos vinculantes de [...] un pacto entre las partes se produzcan sin perjuicio de los derechos de cualquier persona que, en virtud de la ley aplicable a la sucesión, tenga derecho a una parte reservada u otro derecho del que no pueda ser privado por la persona de cuya sucesión se trate" 50 .

45 A. DAVÌ y A. ZANOBETTI, arriba n. 24, p. 68.

46 Para el ordenamiento jurídico francés, véase el Rapport sur la réserve héréditaire publicado en diciembre de 2019 y disponible en: http://www.presse.justice.gouv.fr/art pix/2019.12.20\%20Rapport\%20reserve\%20hereditaire.pdf. Consultado el 29.06.2021.

Para el sistema jurídico italiano, véase M.C. TATARANO, "La successione necessaria", G. PERLINGIERI y R. CALVO (eds.), Diritto delle successioni e delle donazioni, ESI, Nápoles 2015, p. 485 y ss.

47 Sobre este punto concreto, véase A. DAVİ, "Riflessioni sul futuro diritto internazionale privato europeo delle successioni" [2005] Rivista di diritto internazionale, p. 324 ss.

48 A. BUCHER, "Successions", en A. BUCHER (ed) Commentaire romand. Ley sobre el derecho internacional privado. Convención de Lugano, Helbing Lichtenhahn, Bâle 2011, p. 816. Por el contrario, H. KUHN, Der Renvoi im internationalen Erbrecht der Schweiz, Schulthess Polygraphischer, Zúrich 1998, p. 58 y ss.; A. HEINI, "Art. 90”, M. MÜLLER-CHEN y C. WIDMER-LÜCHIRGER (eds.), Zürcher Kommentar zum IPRG, 2a ed., Schultess, Zúrich-Basilea-Ginebra 2004, p. 1067.

49 A. BONOMI, "Successions internationales: conflits de lois et de juridictions" [2010] Recueil des cours 350, p. 253.

50 Ver L. ISOLA, Venire contra factum proprium. Herkun und Grundlagen eines proverbial Rechtsprinzips, Peter Lang Publishing, Frankfurt am Main 2017, p. 10 y ss. 
Parece que la opción política adoptada por el Reglamento sobre sucesiones pretende garantizar una "sobreprotección" de los "herederos forzosos", en consonancia con las tradiciones jurídicas de algunos Estados miembros ${ }^{51}$. Esto parece cuestionable porque es probable que constituya un obstáculo más para la planificación eficaz de la sucesión. También hay que señalar que los tribunales de los países que garantizan una porción de la herencia retenida también han reconsiderado sus posiciones tradicionales ${ }^{52}$.

Este es, por ejemplo, el caso del Tribunal Supremo francés. Incluso antes de la entrada en vigor del Reglamento de Sucesión, ya se habían producido dos casos similares $^{53}$ : dos ciudadanos franceses que llevaban muchos años viviendo en California habían legado todo su patrimonio a sus cónyuges. Intencionadamente, no habían dejado ninguna parte de su patrimonio a sus hijos, como permite la ley de California, que no reconoce la reserva hereditaria.

Los hijos del fallecido iniciaron un procedimiento para obtener su parte de la herencia forzosa. Al final, recurrieron a la Cour de Cassation francesa. Para fundamentar su demanda, se basaron en el llamado "derecho de cobro" (droit de prélèvement) ${ }^{54}$, según el cual los herederos franceses que han sido privados de su parte de los bienes del difunto situados en el extranjero, tienen derecho a cobrar una cantidad igual a la parte de los bienes del difunto situados en Francia.

El Tribunal Supremo francés rechazó este argumento. En particular, afirmó que la ley de California (último lugar de residencia del difunto) era aplicable a la sucesión del patrimonio y precisó además que, en virtud de la ley extranjera designada por la norma de conflicto de leyes, la exclusión de la parte de la herencia forzosa no era, en sí misma, contraria al orden público internacional francés ${ }^{55}$.

En una decisión posterior, el mismo tribunal también reiteró que el fundamento jurídico de la "reserva hereditaria no puede encontrarse en el orden público internacional francés" ${ }^{\prime 56}$.

Superando una larga tradición en sentido contrario ${ }^{57}$, la doctrina y la jurisprudencia italianas han llegado a conclusiones similares.

51 Durante los debates relativos a los trabajos preparatorios del Reglamento sobre la sucesión, el Senado francés adoptó una resolución. Consideró que el principio de la reserva hereditaria era "la traducción jurídica de un verdadero deber moral" y "una norma esencial del Derecho francés", L. RASS-MASSON, "The impact of European Private International Law and the Réserve héréditaire in France", en J.M. SCHERPE y E. BARGELLI (eds.), n. 21, p. 201.

52 A. BONOMI, "Quelle protection pour les héritiers réservataires sous l'empire du future règlement européen", en Droit international privé. Travaux du Comité français de droit international privé 2008-2010, Pedone, París 2011, p. 272.

53 Cass. 27 de septiembre de 2017, números 16-13.151 y 16-17.198, Dalloz 2017, p. 2185. Ver A. BOICHE, "La réserve héréditaire n'est pas d'ordre public international" [2017] L'Actualité juridique: Famille, p. 595 ss.; L. USUNIER, “La reserve héréditaire n'est pas d'ordre public international” [2017] Revue trimestrielle de droit commercial, p. 833 y ss.; H. FULCHIRON, “Ordre public successoral et réserve héréditaire: réflexions sur les notions de précarité et de besoin” [2017] Recueil Dalloz, pp. 2310 y ss.

54 El Consejo Constitucional francés declaró esta ley inconstitucional en 2011: Cons. const., 5 de agosto de 2011, $\mathrm{n}^{\circ}$ 2011-159, véase H. GAUDEMET-TALLON, "Panorama de droit international privé" [2012] Recueil Dalloz, 1228.

55 Ver H. FULCHIRON, arriba n. 52 y A. BOICHE, 'Succession de Johnny Hallyday: le droit international privé saisi par l'actualité people' [2018] L'Actualité juridique: Famille, p. 138.

56 Cass. 4 de julio de 2018, No 17.16.-515 y 17-16.522 [2018] en Juris-Classeur périodique, édition notariale 2018, p. 1313, nota E. Fongaro. Véase también L. RASS-MASSON, "Cour de cassation: Ordre public und Pflichtteilsrecht in Frankreich" [2019] Journal of European Private Law, p. 823.

57 P. GALLO, "Successioni in diritto comparato. Aggiornamento" [2011] en Digesto disciplina pri-vatistiche. Sección Civil, Turín, p. 851 y ss. 
Se ha afirmado con autoridad que las normas de protección de los eredi necessari, aunque sean obligatorias, no aplican derechos humanos inviolables ni otros principios fundamentales en el ordenamiento jurídico italiano ${ }^{58}$. Ello se debe a que la parte reservada no es, per se, funcional a la protección de la persona humana (artículo 2 de la Constitución italiana), sino que, en sentido contrario, puede ser incluso perjudicial en relación con otros principios cuya protección está reconocida y garantizada por la Constitución (como la protección del trabajo, la empresa y el ahorro, artículos 1, 4, 43 y 47 de la Constitución italiana), como reconoció el legislador italiano [...] con la introducción del "patto di famiglia" en 2006 (artículos 768 bis y siguientes del Código Civil italiano) ${ }^{59}$. En consecuencia, al menos en lo que respecta al ordenamiento jurídico italiano, parece correcto afirmar que las normas que protegen la reserva hereditaria no son, en sí mismas, contrarias al orden público internacional ${ }^{60}$, salvo en algunas circunstancias en las que se ven afectadas las razones de solidaridad que subyacen a la reserva hereditaria. A una conclusión similar llegaron también los Tribunales Supremos español ${ }^{61}$ e italiano ${ }^{62}$.

\section{OTRAS LIMITACIONES A LA AUTONOMÍA DE LA VOLUNTAD EN VIRTUD DEL ARTÍCULO 25 DEL REGLAMENTO DE SUCESIONES}

La intención del legislador europeo de no entrar en conflicto con las disposiciones nacionales que protegen la reserva hereditaria no es la única limitación que el Reglamento de sucesiones impone a la eficacia de la planificación contractual de la sucesión.

La norma establecida en el apartado 2 del artículo 25 del Reglamento de sucesiones parece ser especialmente restrictiva en lo que respecta a la autonomía privada. Como se ha señalado, establece que un pacto sucesorio relativo a varias personas sólo es válido si es admisible por todas las leyes que, en virtud de este Reglamento, habrían regido la sucesión de todos los interesados si hubieran fallecido el día en que se celebró el pacto.

58 G. PERLINGIERI y G. ZARRA, Ordine pubblico interno e internazionale tra caso concreto e sistema ordinamentale, ESI, Nápoles 2019, p. 183.

59 G. PERLINGIERI y G. ZARRA, ibídem, p. 184. La ley de 2006 que introduce el "patto di famiglia" pretende proteger a la empresa (artículo 43 de la Constitución italiana) y a los trabajadores (artículos 1 y 4 de la Constitución italiana) durante el relevo generacional. El legislador pretendía evitar el reparto de la empresa entre varios eredi necessari en el momento de su muerte. El "patto di famiglia" es un acuerdo por el que el fallecido transfiere, sin contraprestación, la empresa a un único erede necessario que se compromete a "compensar" a los demás herederos mediante el pago del valor de sus porciones reservadas. Por lo tanto, la parte reservada de los herederos que no obtienen la empresa no es pagada por el difunto.

60 A. DAVÍ y A. ZANOBETTI, supra n. 36, p. 175. Véase también W. PINTENS, "Public Policy in Suc-cession Matters", en Liber Amicorum Kohler, Gieseking Verlag, Bielefeld 2018, p. 395 y ss.

61 Véase Tribunal Supremo, 15 November 1996, Lowenthal, in Revista Española de Derecho Internacional, 1997, p. 264; Tribunal Supremo, 21 May 1999, Denney, ibid.

62 Véase Cass., 24 de junio 1996, n. 5832, en Giustizia civile, 1997, I, p. 1668 sobre la sucesión de un ciudadano italiano (naturalizado canadiense) cuyos herederos forzosos eran ciudadanos italianos, y Cass, 30 de junio 2014, n. 14811, con comentario de E. Calò, "La vedova non è piú allegra: la mancanza di reciprocità con Cuba preclude lo status di legittimario" [2015] Diritto successioni famiglia, p. 567 y ss., que sostenía que la sucesión forzosa no está contemplada en la Constitución, y el legislador bien podría reformarla, o incluso anularla en cualquier momento. El caso se refería a una ciudadana cubana que intentaba recuperar la parte reservada de la herencia de su marido italiano; el Tribunal dictaminó que la demandante no había satisfecho el principio de reciprocidad, dado que la ley cubana no prevé la herencia forzosa. 
Además, el acuerdo se regirá por la ley con la que tenga una conexión más estrecha. Por el contrario, el apartado 2 del artículo 18 de la propuesta de la Comisión se inspiraba en una política más liberal en cuanto a la admisibilidad de los pactos sucesorios celebrados por varias personas: habría sido válido y admisible si estuviera previsto por la ley del Estado (único) en el que una de las partes tuviera su residencia habitual ${ }^{63}$. Sin embargo, se ha afirmado con autoridad que no todos los Estados miembros cuya tradición jurídica es fundamentalmente contraria a la admisibilidad de los pactos sucesorios habrían estado dispuestos a aceptar la solución propuesta por la Comisión ${ }^{64}$.

Sin embargo, hay que matizar la idea de que los Estados mencionados se oponen radicalmente a los pactos sucesorios. Es cierto que todavía hay muchos países que prohíben los pactos por los que el testador organiza su propia sucesión en vida: Francia, Bélgica, Luxemburgo (artículo 1130, apartado 2, del Código Civil), Países Bajos (artículo 4.4, apartado 2, de la NBW), Portugal (artículo 2028, apartado 2, del Código Civil), Grecia (artículo 368 del Código Civil), España (aunque se prevén excepciones a nivel regional) ${ }^{65}$ e Italia (artículo 458 del Código Civil). Sin embargo, un examen más detallado muestra que esta prohibición no carece de excepciones.

En Francia, por ejemplo, la jurisprudencia se ha pronunciado a favor de la admisibilidad de los pactos sucesorios ${ }^{66}$. Por otra parte, el legislador francés aprobó la Ley $\mathrm{n}^{\mathrm{o}} 2006-728$, de 23 de junio de 2006, con el fin de ampliar los espacios reservados a la autonomía privada y acelerar la liquidación de la herencia ${ }^{67}$. En su redacción actual, el Código Civil francés mantiene la norma general que prohíbe los pactos sobre sucesiones futuras (artículos 722, 791, 943, 1389, 1600, 1837). Al mismo tiempo, sin embargo, establece un régimen de excepciones para los (no tan pocos) casos previstos por la ley ${ }^{68}$. Hoy en día existen numerosos ejemplos de acuerdos típicos en materia de sucesión, como la cláusula comercia ${ }^{69}$ y la donation-partage ${ }^{70}$. La doctrina jurídica, en efecto, se pregunta si la prohibición no se ha perdido, en el fondo, por completo ${ }^{71}$.

63 Artículo 18, apartado 2, de la Propuesta de Reglamento del Parlamento Europeo y del Consejo relativo a la competencia, la ley aplicable, el reconocimiento y la ejecución de las resoluciones y los actos auténticos en materia de sucesiones y a la creación de un certificado sucesorio europeo \{SEC(2009) 410\} \{SEC (2009)411\}: Un pacto relativo a la sucesión de varios hijos sólo será válido en cuanto al fondo si esta validez es aceptada por la ley que, en virtud del artículo 16, se habría aplicado a la sucesión de una de las personas de cuya sucesión se trate en caso de fallecimiento el día en que se celebró el pacto".

64 A. DAVÍ y A. ZANOBETTI, arriba n. 36, p. 178.

65 MARTINEZ M., "Les pactes successoraux dans les droits régionaux d'Espagne”, en A. BONOMI, M. STEINER (eds.), Les pactes successoraux en droit comparé et en droit international privé, Libraire Droz, Ginebra 2008, p. 107 y ss.

66 Con referencia a Francia, véase Cass. 30 de mayo de 1985, en J Dalloz, 1986, p. 65 y ss.; Cour d'appel Aix-enProvence, 16 de octubre de 2003, en Revue critique droit internation privé, 2004, p. 589 y ss.

67 PH. MALAURIE, "Examen critique du projet de loi portant réforme des successions et des libéralités" [2005] Defrenois, 38298; D. VIGNEAU, "Le règlement de la succession. Observation sur le projet de loi portant réforme des successions et des libéralités', [2006], Juris-Classeur périodique, édition notariale, p. 1144; A.M. LEROYER, 'Reforme des successions et des libéralités. Loi n. 2006-728 of 23 June 2006" [2006] Revue trimestrelle droit civil, p. 613.

68 A. BRAUN, "Towards a Greater Autonomy for Testators and Heirs: Some Reflections on Recent Reforms in France, Belgium and Italy" [2012] Zeitschrift für Erbrecht und Vermögensnachfolge, p. 461 y ss.

69 F. XAVIER TESTU, "Pactes sur succession future exceptionnellement autorisés", en M. GRIMALDI (ed.), Droit patrimonial de la famille, Dalloz, París 2009, p. 395 y ss.

70 A.M. LEROYER, n. 66 anterior.

71 N. BAILLON-WIRTZ, "Que reste-t-il de la prohibition des pactes sur succession future?" [2006] Droit de la famille, pp. 8 y ss. 
Incluso la doctrina italiana más autorizada ha sostenido, además, que un pacto sucesorio admitido sobre la base de una ley extranjera elegida en virtud del artículo 25 , apartado 2, del Reglamento de sucesiones puede considerarse admisible y válido: según esta perspectiva, la entrada en vigor del Reglamento de sucesiones significa que la validez y la eficacia del pacto sucesorio no deben medirse con prohibiciones internas abstractas que, por sí mismas, no pueden impedir su aplicación ${ }^{72}$.

Los problemas de aplicación práctica que se derivan del apartado 2 del artículo 25 pueden verse mitigados por las disposiciones del apartado 3 del artículo 25 del Reglamento de sucesiones ${ }^{73}$. La diferencia entre ambos es clara. Se ha descrito como una verdadera "contradicción interna" en el Reglamento de sucesiones, como ocurrió con los artículos 10 y 11 del Convenio de La Haya ${ }^{74}$.

Sin embargo, si se examina más detenidamente, incluso el apartado 3 del artículo 25 puede no ser útil para la planificación de la sucesión. No impide una división entre la ley aplicable a la sucesión (en virtud de los artículos 21 y 22 del Reglamento sobre sucesiones) y la ley que rige el pacto sucesorio. Además, su aplicación corre el riesgo de vulnerar el principio de unidad de la sucesión. Sólo queda esperar, como ya se ha dicho, que "las partes ejerzan esta autonomía con prudencia"

\section{RETOS DE LA APLICACIÓN PARALELA DEL REGLAMENTO DE SUCESIONES Y DE LOS REGLAMENTOS GEMELOS}

En este contexto, la identificación de la ley aplicable a una pareja transfronteriza casada o registrada que desea organizar su planificación sucesoria parece algo problemática. Se trata de una cuestión muy delicada, ya que la calificación implica una interpretación sistemática en la que intervienen el Reglamento sobre el régimen económico matrimonial y el Reglamento sobre los efectos patrimoniales de las uniones registradas (en adelante, el Reglamento gemelo), que se aplican actualmente en 18 Estados miembros $^{76}$.

El artículo 22 de los Reglamentos gemelos permiten a los cónyuges y a las parejas de hecho registradas elegir la ley del Estado en el que al menos uno de ellos reside o la ley de la nacionalidad de uno de ellos, o bien, para las parejas registradas, la ley del Estado bajo cuya ley se creó la unión registrada. Por lo tanto, según la naturaleza del acuerdo, el papel de la autonomía de la voluntad puede ser más o menos relevante ${ }^{77}$ : el escaso margen

72 G. PERLINGIERI y G. ZARRA, n. 57, p. 198. Sobre este tema, con referencia al sistema jurídico italiano, véase también C. CACCAVALE, Contratto e successione, en V. ROPPO (ed.), Trattato dei contratti, vol. VI, Giuffré, Milán 2006, p. 403 ss. y V. PUTORTÍ, Il divieto dei patti successori alla luce del Regolamento UE 650/2012" [2016] Diritto Successioni Famiglia, p. 845 y ss.; V. BARBA, n. 32 supra, p. 10 y ss.

73 El Bundesgerichtshof alemán aplica esta disposición a un pacto sucesorio celebrado antes de la entrada en vigor del Reglamento de sucesiones, véase BGH, 10 de julio 2019, IV ZB 22/18, Zeitschrift für Das Gesamte Familienrecht 2019, p. 1561, comentado por Von Bar.

74 A. DAVÍ y A. ZANOBETTI, n. 36 supra, p. 183.

75 M. PAZDAN y M. ZACHARIASIEWICZ, n. 9, p. 134.

76 Nueve Estados miembros siguen al margen de la cooperación reforzada. En relación con estos Estados, véase A. WYSOCKA-BAR, "Enhanced cooperation in property matters in the EU and non-participating Member States" [2019] ERA Forum 20, p. 187 y ss.

77 P.R. WAUTELET, 'What's Wrong with Article 22? The Unsolved Mysteries of Choice of Law for Matrimonial Property' [2018] disponible en https://ssrn.com/abstract=3266879. Consultado el 29.06.2021. 
de maniobra que concede la ley que regula los acuerdos similares en materia de sucesión ${ }^{78}$ muestra claramente la distinción con respecto a los acuerdos patrimoniales "relacionados con la pareja", para los que la posibilidad de elegir la ley aplicable en virtud de la normativa de la UE es más amplia ${ }^{79}$.

Un problema muy importante es el de los pactos entre cónyuges o parejas en relación con la planificación de la herencia o que tienen efectos sobre ella. ¿Debe aplicarse el Reglamento sobre sucesiones o, alternativamente, los Reglamentos gemelos y, en consecuencia, ¿qué ley será aplicable?.

Muchos casos corren el riesgo de caer en esta "zona gris" debido a los problemas descritos de coordinación entre los reglamentos. Es el caso, por ejemplo, de los "contratos sucesorios" con efectos post mortem (el Erbvertrag alemán ${ }^{80}$, artículo 2264 del Bürgerliches Gesetzbuch y el pacto sucesorio austriaco según los artículos 1249 y siguientes del Allgemeines Bürgerliches Gesetzbuch) y el Berliner Testament según el artículo 2265 del BGB. Según estas disposiciones, los cónyuges y las parejas de hecho se erigen en herederos mutuos. Asimismo, establecen que la totalidad de la herencia tras el fallecimiento del último en morir debe recaer en los hijos de la pareja. Ejemplos similares en el ordenamiento jurídico francés son la liberalités-partages y la clause commerciale ${ }^{81}$.

Teóricamente, la línea divisoria entre el Reglamento de sucesiones y los Reglamentos gemelos es muy clara. En efecto, mientras que la letra d) del apartado 2 del artículo 1 del Reglamento de sucesiones excluye los bienes matrimoniales de su ámbito de aplicación material, la letra d) del apartado 2 del artículo 1 de los Reglamentos gemelos excluye las sucesiones. Sin embargo, los ejemplos anteriores muestran que, en concreto, el trazado de los límites entre ambos instrumentos depende en gran medida del significado exacto de "sucesión", por un lado, y de "régimen económico matrimonial", por otro ${ }^{82}$.

La cuestión práctica que se deriva de esta falta de coordinación es que la ley aplicable en virtud del artículo 22 de los Reglamentos gemelos puede diferir de la aplicable a una determinada sucesión en virtud del Reglamento sobre Sucesiones. Dos leyes diferentes regirán los bienes matrimoniales y la sucesión cuando, en el momento del fallecimiento de uno de los cónyuges o convivientes, éste tuviera su residencia habitual en un país distinto al de la primera residencia habitual después del matrimonio. Esto ocurre sobre todo cuando una pareja transfronteriza ha cambiado su residencia habitual común durante el matrimonio o la pareja. Esto supondría claramente un obstáculo adicional para la planificación sucesoria de las parejas ${ }^{83}$.

78 Véanse los apartados 3 y 4 anteriores.

79 Véase E. BERGAMINI, "Agreements between spouses and partners, and agreements as to successions", en S. LANDINI (ed), EU Regulations 650/2012, 1103 and 1104/2016: cross-border fami-lies, international successions, mediation issues and new financial assets, ESI, Nápoles 2020, p. 106.

80 E.A. AMAYUELAS, The Law of succession. Testamentary Freedom, Groningen, European Law Publishing 2011, p. 165 y ss. y H. BROX y W. WALKER, Erbrecht, $28^{a}$ ed., Beck, Vahlen, 2018, p. 10 y ss.

81 Véanse, más arriba, las secciones 3 y 4.

82 Como señala A. BONOMI, "The Interaction Among the Future EU Instruments on Matrimonial Property, Registered Partnerships and Successions" [2011] Yearbook Private International Law 13, p. 219 y ss.

83 A. BONOMI, "The Regulation on Matrimonial Property and Its Operation in Succession Cases - Its Interaction with the Succession Regulation and Its Impact on Non-participating Member States" [2020] Problemy Prawa Prywatnego Międzynarodowego 26, p. 85. 
El TJUE abordó esta cuestión en el caso Mankopf ${ }^{84}$, un litigio relativo a la Zugewinngemeinschaft alemana ${ }^{85}$. El Sr. Mahnkopf estaba casado con la Sra. Mahnkopf. Eran ciudadanos alemanes con residencia habitual en Berlín. El Sr. Mahnkopf falleció el 29 de agosto de 2015 sin haber hecho ninguna disposición de bienes para después de su muerte: los únicos herederos eran su esposa y su único hijo. El Sr. y la Sra. Mahnkopf no habían celebrado ningún contrato matrimonial. En consecuencia, estaban sujetos al régimen patrimonial legal de comunidad de ganancias acumuladas. La herencia incluía una media participación en la copropiedad de un inmueble en Suecia.

La mitad de la herencia de la Sra. Mahnkopf resultaba de la aplicación del artículo 1931(1) del BGB, según el cual la cuota del cónyuge superviviente en la sucesión, que era de una cuarta parte, se incrementaba en una cuarta parte adicional cuando ambos cónyuges estaban sujetos al régimen económico matrimonial de la comunidad de ganancias acumuladas. La viuda solicitó un certificado sucesorio europeo en Alemania para registrar una herencia en Suecia ${ }^{86}$. Y el notario presentó la solicitud de la Sra. Mahnkopf al tribunal local. Este último rechazó la solicitud porque la parte asignada al cónyuge del difunto se basaba (en lo que respecta a una cuarta parte del patrimonio del difunto) en un régimen de sucesión y (en lo que respecta a otra cuarta parte de su patrimonio) en el régimen económico matrimonial previsto en el artículo 1371, apartado 1, del BGB. En su opinión, la norma en virtud de la cual se asignó la otra cuarta parte, que se refiere a un régimen económico matrimonial y no a un régimen sucesorio, no entra en el ámbito de aplicación del Reglamento sobre sucesiones. En este caso, se planteó la cuestión de si el ámbito de aplicación del Reglamento sobre sucesiones incluye también las disposiciones relativas a los regímenes matrimoniales que dan lugar a un aumento de la cuota de uno de los cónyuges o de la pareja tras el fallecimiento del otro cónyuge o de la pareja.

El TJUE no analizó si esta disposición tiene carácter matrimonial o sucesorio en el derecho alemán, cuestión enmarañada y muy debatida en la teoría del derecho privado alemán ${ }^{87}$. Más bien, el Tribunal hizo un análisis objetivo y orientado a la función de la cuestión. Señaló que la disposición legal no trataba de la distribución de los bienes entre los cónyuges o parejas, sino que operaba una vez que la distribución había tenido lugar, y sólo después de la muerte. En consecuencia, afirmó que “a efectos del Reglamento”, el artículo 1371, apartado 1, del BGB es aplicable en materia de sucesión.

84 C-558/16 Mahnkopf, ECLI:EU:C:2018:138 en la que el tribunal dictaminó que una disposición como el artículo 1371, apartado 1, del BGB "que prescribe, a la muerte de uno de los cónyuges, una alocación fija de las ganancias acumuladas mediante el aumento de la cuota hereditaria del cónyuge supérstite está comprendida en el ámbito de aplicación de dicha normativa”. J. WEBER, "Ein Klassiker neu aufgelegt: Die Qualifikation des §1371 BGB unter dem Regime der Europäischen Erbrechtsverordnung” (2018) Neue Juristische Wochenschrift, p. 1357.

85 Véase I. BARRIÈRE BROUSSE, "Conflit de lois" [2018] Journal du droit international 4, p. 1218 y ss. Para una evaluación crítica del caso, véase J. VON HEIN, 'The CJEU settles the issue of character-ising the surviving spouse's share of the estate in the context of the Succession Regulation' [2018] disponible en conflictoflaws.net. Consultado el 29.06.2021.

86 Para una evaluación de la correlación entre las relaciones familiares y el ECS, véase D. DAMASCELLI, "Brevi note sull'efficacia probatoria del certificato successorio europeo riguardante un soggetto coniugato o legato da unione non matrimoniale" [2017] Rivista di diritto internazionale pro-cessuale, p. 67 y ss.

87 Véase T. JÄGER, "Der neue Güterstand der Wahl-Zugewinngemeinschaft. Inhalt und seine ersten Folgen für die Gesetzgebung und Beratungspraxis" [2010] Deutsche Notar-Zeitschrift, p. 804 y ss. y W. PINTENS, Ehegüterstände in Europa, en Lipp-Schumann-Veit (ed.), Die Zugewinngemeinschaft. Ein europäisches Modell?, Universitätsverlag Göttingen, Göttingen 2009, p. 23. 
Según la interpretación del TJUE ${ }^{88}$, las normas nacionales entran en el ámbito de la "sucesión" cuando "despliegan sus efectos en el caso de la sucesión" y "determinan los derechos del cónyuge supérstite en la relación con los demás herederos" $"$. Hay que señalar que, dada la diversidad de tradiciones jurídicas nacionales, el criterio utilizado por el TJUE es, de nuevo, la función del acto concreto ${ }^{90}$. Es precisamente esta regla de interpretación la que debe utilizarse para superar la antinomia descrita anteriormente. El TJUE no entra en el análisis del fundamento de la voluntad de las partes ni de las tradiciones jurídicas nacionales. Por el contrario, califica los casos concretos en relación con la finalidad objetiva de los acuerdos entre cónyuges o parejas.

Sobre esta base debe resolverse la antinomia: si un acuerdo entre cónyuges y parejas despliega sus efectos en materia de sucesión y determina los derechos del cónyuge supérstite en relación con los demás herederos, debe regirse por el artículo 25 del Reglamento de sucesiones.

Este razonamiento puede aplicarse también en casos similares. A pesar de su naturaleza de acuerdo entre cónyuges, por ejemplo, la cláusula comercial debe calificarse, "a los efectos del Reglamento", como disposiciones por causa de muerte (y más concretamente como acuerdos en materia de sucesión, en el sentido de la letra b) del apartado 1 del artículo 3 del Reglamento de sucesiones). En consecuencia, aunque se trate de una institución tradicionalmente vinculada al régimen económico matrimonial, la norma de conflicto no entraría en el ámbito de aplicación del artículo 22 de los Reglamentos gemelos.

La misma solución puede analizarse en los casos similares antes descritos si, funcionalmente, producen los efectos vistos en Mankopf, siendo un ejemplo las "donaciones entre cónyuges" francesas (institution contractuelle) ${ }^{91}$.

El problema de interpretación se habría superado si el Reglamento de sucesiones hubiera permitido utilizar la ley de la residencia habitual de una de las partes implicadas como ley aplicable al acuerdo. De este modo, las dificultades para distinguir los diferentes tipos de acuerdos habrían sido menos significativas: "habría sido posible que las parejas hicieran una elección de ley válida en virtud de ambos Reglamentos [de los gemelos y de la sucesión] que pudiera abarcar todos los tipos de acuerdos recíprocos si se consideraran relacionados con la sucesión o con los bienes matrimoniales/de pareja" ${ }^{92}$.

Sin embargo, en el plano de la aplicación práctica, sigue existiendo el problema de la orientación que debe darse a las parejas transfronterizas que desean planificar su sucesión, directamente o no. Los juristas han señalado, con razón, que la coordinación entre el artículo 22 de los Reglamentos gemelos y los artículos 3 y 25 del Reglamento sobre sucesiones puede ser realizada por los cónyuges o las parejas registradas mediante la elección de la ley aplicable ${ }^{93}$. En particular, si cambian de residencia habitual, pueden someter su

88 Y, en un sentido similar, también el asunto del TJUE, C-218/16, Kubicka, ECLI:EU:C:2017:755. Sobre este punto, véase W. BAŃCZYK, "The efficiency of the foreign legacy 'by vindication' in a state not recognising it and the borders of succession law, based on Regulation (EU) No 650/2012" [2020] Zeitschrift für europäisches Privatrecht, pp. 710-728.

89 Asunto C-558/16 Mahnkopf, ECLI:EU:C:2018:138, párr. 40.

90 Sobre la complejidad y la unidad funcional del sistema jurídico actual véase P. PERLINGIERI, Il dirit-to civile nella legalità costituzionale secondo il sistema italo-europeo delle fonti, vol. 2, $4^{\mathrm{a}}$ ed., ESI, Nápoles 2020, p. 59 y ss.

91 Ver D. DAMASCELLI, Diritto internazionale privato delle successioni, $\mathrm{n}^{\circ} 42$ supra, p. 93 y ss.

92 E. BERGAMINI, $\mathrm{n}^{\circ} 79$ anterior, p. 107.

93 A. BONOMI, $\mathrm{n}^{\circ} 83$ anterior, p. 86. 
relación matrimonial a la ley de su nueva residencia habitual. Esta elección será útil si es la ley aplicable a su sucesión. Si aceptan que su elección tenga un efecto retroactivo, una sola ley podrá aplicarse a todas estas cuestiones ${ }^{94}$.

\section{OBSERVACIONES FINALES: ¿A QUÉ CONCLUSIONES PODEMOS LLEGAR DESPUÉS DE LO EXPUESTO ANTERIORMENTE?}

Está claro que la evolución del derecho en lo que respecta al reconocimiento de los efectos legales de las relaciones es muy dinámica, tanto en el plano sustantivo como en el del derecho internacional privado. A lo largo de la última década, el mapa de Europa ha cambiado de forma constante y sigue desplazándose a gran velocidad ${ }^{95}$.

La europeización de las normas de conflicto parece haber aflojado los vínculos tradicionales que las normas de Derecho Internacional Privado tenían con las leyes nacionales $\mathrm{y}$, en particular, con la nacionalidad ${ }^{96}$. Al mismo tiempo, sin embargo, esta fragmentación no parece haber roto el vínculo con el derecho sustantivo ${ }^{97}$.

A diferencia de otros ámbitos, en los que la armonización ha sido inmediata y directa (por ejemplo, el derecho de los consumidores), la europeización de las normas de conflicto ha consolidado y reforzado el diálogo entre los tribunales ${ }^{98}$, requisito esencial para la creación del futuro derecho privado europeo ${ }^{99}$. En este contexto, la DPI exige a los juristas nacionales y a los profesionales del Derecho que tengan en cuenta los sistemas jurídicos de los demás Estados miembros ${ }^{100}$ para desarrollar un espacio de libertad, seguridad y justicia en el que se garantice la libre circulación de todas las personas ${ }^{101}$.

Se trata de un resultado muy satisfactorio, especialmente en relación con un ámbito jurídico con un fuerte componente tradicional como es la familia y la herencia ${ }^{102}$. Sin embargo, la importancia de la LIP como motor del proceso de integración europea corre el riesgo de verse gravemente limitada por las disposiciones incoherentes de la legislación

94 A. BONOMI, ibídem, p. 85.

95 W. SCHRAMA, "Empowering private autonomy as a means to navigate the patchwork of EU Regulations", en J.M. SCHERPE y E. BARGELLI (eds.), n. 21, p. 55.

96 M. PERTEGAS SENDERS y M.C. BOGDAN, Concise Introduction to EU Private International Law, $4^{\mathrm{a}}$ ed., Groeningen, Europa Law Publishing, 2019, p. 13 y ss.

97 Véase J. BASEDOW, "The Communitarisation of Private International Law" [2009] Rabels Zeitschrift für ausländisches und internationales Privatrecht 73, p. 455 y ss.

98 Ver D. ACHILLE, "Lex successionis e compatibilità con gli ordinamenti degli stati membri nel Reg. UE n. 650/2012" [2018] Nuova giurisprudenza civile commentata, p. 697 y ss.

99 A. GAMBARO y R. SACCO, "Sistemi giuridici comparati", en R. SACCO (ed.), Trattato di diritto comparato, UTET, Turín 2008, p. 27 y ss. Véase también S. DEPLANO, "Verso un codice dei contratti europeo?” [2010] Rivista giuridica Molise e Sannio, p. 105 y ss.

100 Para un ejemplo en el ámbito del Derecho de familia, véase M.J. CAZORLA GONZÁLEZ, M. GIOBBI, J. KRAMBERGER ŠKERL, L. RUGGERI y S. WINKLER (eds.), n. 7 supra, p. 14 y ss.

101 P. BENVENUTI, "Il diritto internazionale privato", en C. CASTRONOVO y S. MAZZAMUTO (eds.), Manuale di diritto internazionale privato, vol. 1, Giuffré, Milán 2007, p. 125 y ss., donde se afirma que el Derecho internacional privado permite relativizar, por vía de integración, la pertenencia de cada sistema de Derecho privado a un ordenamiento jurídico estatal originario.

102 C. CONSOLO y F. GODIO, "Profili processuali del Reg. UE n. 650/2012 sulle successioni transnazionali: il coordinamento tra giurisdizioni" [2018] Rivista di diritto civile, p. 18 y ss. 
de la $\mathrm{UE}^{103}$. Un papel central en la superación de estas incoherencias, quizás inevitable a la vista de la complejidad de la legislación, lo desempeñan las teorías jurídicas nacionales y el TJUE, que está asumiendo un papel cada vez más central en el proceso de interpretación ${ }^{104}$. Sería conveniente que el TJUE siguiera contribuyendo a facilitar y armonizar la aplicación de los regímenes de LIF en los Estados miembros de la UE ${ }^{105}$.

103 V. HEINZE, “The European Succession Regulation n. 650/2012. An Overview”, en G. ALPA (ed.) "I nuovi confini del diritto privato europeo. Nuove frontiere del diritto privato europeo", Giuffré, Milán 2016, p. 45: "El Derecho internacional privado de la UE no se promulgó como una codificación global, sino que procede por etapas, cada una relacionada con un ámbito sustantivo específico".

104 Sobre el papel del TJUE en el proceso de unificación, en particular del Derecho privado europeo, véase I. KLAUER, Die Europäisierung des Privatrechts, Baden Baden, Nomos 1998; J. SMITS, The making of European Private Law. Towards a Ius Commune Europaeum as a Mixed Legal System, Intersentia, Amberes, 2002, p. 19 y ss. y C. TWIGG-FLESNER, The Europeanisation of Contract Law. Current controversies in law, $2^{\mathrm{a}}$ ed., LondresNueva York, Taylor \& Francis, 2013.

105 Sobre la relación entre los tribunales nacionales e internacionales en el sistema jurídico actual, véase P. FEMIA, "Decisori non gerarchizzabili, riserve testuali, guerra tra Corti. Con un (lungo) intermezzo spagnolo", en V. RIZZO y L. RUGGERI (eds.), Il controllo di legittimità costituzionale e comunitaria come tecnica di difesa, ESI, Nápoles 2010, pp. 85-270. 